\title{
Téoros
}

Revue de recherche en tourisme

\section{La délégation de gestion dans les parcs du Québec : pour un partenariat efficace}

\section{Serge Robillard}

Volume 14, numéro 1, printemps 1995

Les parcs : des réseaux en mutation

URI : https://id.erudit.org/iderudit/1077050ar

DOI : https://doi.org/10.7202/1077050ar

Aller au sommaire du numéro

Éditeur(s)

Université du Québec à Montréal

ISSN

0712-8657 (imprimé)

1923-2705 (numérique)

Découvrir la revue

Citer cet article

Robillard, S. (1995). La délégation de gestion dans les parcs du Québec : pour un partenariat efficace. Téoros, 14(1), 17-19. https://doi.org/10.7202/1077050ar d'utilisation que vous pouvez consulter en ligne.

https://apropos.erudit.org/fr/usagers/politique-dutilisation/ 


\title{
La délégation de gestion dans les parcs du Québec : pour un partenariat efficace
}

\author{
Serge Robillard*
}

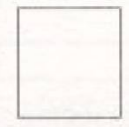

Ala finde 1993, le gouvernement libéral del'époqueannonçit son intention d'amorcer un vaste processus de privatisation des parcs, et par là sa volonté de procéder à une délégation totale de ses responsabilités et activités dans les parcs et réserves du Québec. Il n'en fallait pas moins pour susciter un débat public qui eut des échos dans bon nombre de régions au Québec. Les réactions nombreuses amenèrent le gouvernement à annoncer un moratoire sur l'ensemble du projet.

Suite à un remaniement ministériel qui confia ledossier des parcs et des réserves au ministère de l'Environnement et de la Faune, monsieur Paradis, ministre responsable de ce secteur, annonçait que le gouvernement allait maintenir son objectif de déléguer la gestion des activités commerciales dans les parcs du Québec, mais que l'État se réservait les responsabilités lices aux orientations et à la protection du patrimoine naturel, l'accueil des usagers et les activités d'interprétation.

\section{Le projet de délégation de gestion dans les parcs du Québec}

Dernièrement, le gouvernement du Parti québécois, par son ministre responsable, monsieur Brassard, annonçaitsonintention de poursuivre dans le même sens, et par la même occasion, ouvrait la porte à l'éventualité que des droits d'entrée puissent être imputés aux usagers des parcs du Québec.

Il est donc assez clair, en analysant les déclarations gouvernementales, tant de l'ancien gouvernement quedel'actuel, que la démarche de délégation dans laquelle veuts'orienter l'Étatne vise pasàsedépartir des actifs patrimoniaux que sont les parcset les réservesdu Québec, nià abandonner ses responsabilités, voire sa juridiction, en la matière, ce qui serait, le cas échéant, une privatisation pure et simple.

Le projet de délégation n'envisage que de confier à des tiers la prestation d'activités et de services afin de réduire les déficits d'exploitation, participant ainsi, dans le secteur des parcs, à l'objectif de réduction des dépenses globales de l'État.

D'entrée de jeu, disons que cette approche n'estpas nouvelle. Depuisplusieursannées l'Etat confie, par délégation de gestion, la prestation de plusieurs activités et services aux usagers dans ses parcs. C'est le cas, par exemple, du parc d'Oka qui est géré depuis 1991 par un organisme issu du milieu, mais qui l'a été auparavant par l'entreprise privée: Sodem (de 1986 à 1989) et Sphinx en 1989-1990. C'est également le cas du parc du Mont-Orford, dont bon nombre d'activités et de services aux usagers sont gérés par Soroma, une filiale du groupe Sodem. Sodem est également impliquée dans la prestation d'activités et de services dans les parcs de la Yamaska et des Iles-deBoucherville.

L'expérience de délégation de gestion vécue par Sodem dans le domaine des parcs, depuis près de douze ans maintenant, a beaucoup évolué au cours des années. De simple prestataire de services aux usagers dans les parcs, sous forme de contrats d'impartition, l'entreprise est devenue, au cours des années, un partenaire concessionnaire de l'État. Elle a été appelée à prendre des risques et à réinvestir une partie importante de ses redevances pour contribuer à l'effort d'amélioration des installations et des serviceset cela, en acoord avec les orientations del'Etat et en réponse aux besoins du milieu. C'est le cas du parc du Mont-Orford où près de $500000 \mathrm{~S}$ auront été réinjectés dans le parc pour les années 1993,1994 et 1995.

La délégation de gestion dans les parcs du Québec est appelée encore à évoluer. Elle s'inscrit maintenant, à notre avis, dans le discours dela décentralisation. Unevolonté collective s'exprime de rapprocher le pouvoir décisionnel le plus près possible des milieux et des citoyens. L'objectif visé est de faire participer le milieu aux décisions d'orientation, de l'impliquer et de le responsabiliser davantage dans le processus de choix collectif qui s'impose dans les services à offrir aux citoyens. 
Dans cette perspective, et şns que le rôle et les responsabilités de l'État dans le domaine des parcs soient remis en cause, il en va de l'importance du maintien au Québec d'un réseau de parcs, la question se posesur les modèles de délégation de gestion à mettre en place. À notre avis, deux défis se présentent actuellement à l'Etat: s'associer le milieu dans le choix des orientations visant à définir, planifier et améliorer la prestation d'activités et de services aux usagers des parcs et déléguer la gestion des opérations de ces services et activités à un tiers en visant et en s'assurant d'une prestation de services de qualité et d'une réduction des coûts d'exploitation. Le ou les modèles de délégation de gestion qu'adoptera l'État québécois devront répondre à ces deux défis principaux.

\section{Un modèle de délégation de gestion}

Plusieurs options se présentent ì l'État danslesquellesl'entreprise privée peutjouer un rỏle important. Cette dernière a démontré dans bien des domaines son efficacité et sa performance en ce qui a trait à la prestation d'activités et de services. Son esprit d'entrepreneur en fait un partenaire privilégié des services publics pour investir et développer là où ce dernier peut de moins en moins le faire. Ses objectifs de rentabilité la conduisent naturellement à viser un haut degré de satisfaction des clientèles, à améliorer constamment ses produits et ses services et à créer tous les maillages possibles avec les organismes et les entreprises du milieu afin de per former plus efficacement, compte tenu, particulièrement de l'importance qu'occupe les parcs du Québec dans le développement touristique des régions.

Il n'estcependantpaşdu rôledel'entreprise de se substituer à l'État ou au milieu dans l'exercice des rôles qui doivent leur être dévolus; les choix d'orientation, notarnment, doivent leur appartenir, comptetenu de leur rôle de protecteur du patrimoine public. Dans le contexte des objectifs visés par la délégation de gestion dans les parcs du Québec, l'entreprise peut y jouer un rôle de délégataire-opérateur efficace, mais ne peut y jouer un rôle de décideur sur le plan des orientations, que ce soit dans le domaine des vocations des sites à définir, le choix des activités et des services à offrir ou les politiques à mettre en application. L'entreprise peut collaborer et participer

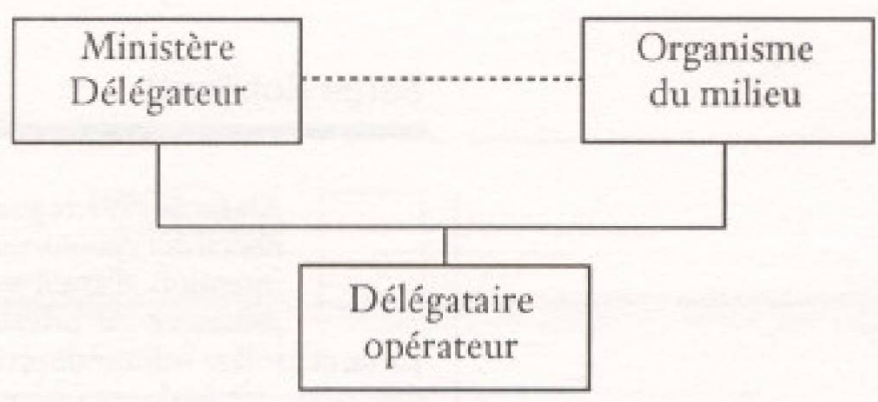

lien fonctionnel

lien d'autorité sur les responsabilités appartenant au milieu

évidemment à de telles prises de décision en y apportant son expertise, mais il ne lui appartient pas ultimement de décider de ces questions. Ce rôle appartient, à notre avis, à l'Etat et, dans une volonté de décentralisation, au milieu et aux mécanismes qu'il se sera donnés pour agir à titre de partenaire de l'Etat. La figure suivante illustre le modèle de délégation de gestion que l'État pourrait mettre en place pour atteindre les objectifs visés et dans lequel l'entreprise privée, comme délégataireopérateur, peut jouer un rôle.

Dans ce modèle, l'État, par le biais de son ministère, conserve ses prérogatives en tant que propriétaire et gardien du patrimoine, législateur et décideur ultime en matière d'orientations et de choix des fonctions, notamment sur le plan de la prestation des services et des activités aux usagers, qu'il entend déléguer.

Dans une volontédedécentralisation, l'État pourrait partager ses prérogatives avec un organisme représentatif du milieu et ce, dans les limites d'un partenariat à établir. Dans cette perspective, et sans définir ce qui est du ressort du milieu, nous croyons que le mandat général de cet organisme devrait être de s'assurer que la gestion et le développement du parc se réalisent en harmonie aveclesintétêts de la région. Cet organisme pourrait être aussi un canal important par lequel pourraient être dirigées toutes les ressources disponibles et vouées au développement du parc.

Quant au délégataire-opérateur, il se voit confier la gestion des opérations relatives à l'offre d'activités et de services. Quelque soit la nature de l'organisme délégataire (entreprise privée, organisme à but non lucratif ou autres) son choix doit se faire à partir de sa capacité à réaliser la mission pour laquelle il sera mandaté.

Dans le modèle que nous présentons, les fonctions d'orientation de conservation et de développement et celles d'opération sont nettement distinctes et appartiennent à des organismes différents. Bien qu'il soit évident que les frontières entre ces deux niveaux de fonctions ne sont pas étanches, il nous apparait important que la division des rôles soit entretenue. Chacun des intervenants pourront et devront se concentrer sur leur mission propre.

Ce modèle, croyons-nous à l'avantage d'associer des partenaires, de partager les responsabilités et de permettre de relever les défis de la décentralisation et d'une gestion déléguée efficace.

Il existe cependant des conditions à respecter pour que la délégation de gestion à un opérateur, dans un esprit de partenariat, soit efficace et ce, quelque soit la nature et le statut de l'organisme délégataire.

\section{Pour un partenariat efficace}

La clé de voûte d'un partenariat réussi, en matière de délégation de gestion, c'est la volonté agissante de développer et d'exercer, tout au long du processus, un véritable partenariat dont nous vous présentons les deux facettes que nous jugeons les plus importantes: le respect des objectifs des 
partenaires et le respect du partenaire dans l'action.

\section{Le respect des objectifs des partenaires}

Cette volonté doit se manifester dès la préparation d'un projet de partenariat de la part de l'instance publique ou gouvernementale. D'abord, en explicitantclairement et de façon tangible (mesurable) tous les objectifs qui sont poursuivis par le délégateur et qui feront, bien sûr, l'objet principal des engagements contractuels.

Cette clarification des objectifs permettra au délégateur de déterminer le type de partenaire souhaité, avec lequel il peut a priori le mieuxy travailler: un organisme à but lucratif ou à but non lucratif, un organisme ayant une expertise privilégiée dans un secteur d'activités déterminé, ete. Il n'y a pas, à ce sujet, de formule parfaite ni de modèle idéal, chaque situation commande une analyse spécifique et une réponse adaptée.

La recherche de ce partenaire, qui peut se faire de différentes façons (appel d'offres, préqualification des partenaires privés éventuels, négociations de gré à gré), requiert de la vigilance et ne doit pas venir contrecarrer les objectifs poursuivis. [] importe d'avoir à l'esprit que, plus le partenaire recherché sera associé au développement de la ressource ou du service, plus grandes seront les exigences et plus les moyens de recherche de ce partenaire devtont être resserrés.

Cela aura des conséquences importantes pour la suite des relations entre les partenaires, car il devra y avoir de partet d'autre, tout au long de la relation de partenariat, un respect intégral de la nature des partenaires.

Le partenaire privé qui s'engage dans une relation avec un délégateur public doit en connaître et en assumer les objectifs instrtutionnels et opérationnels. On a souvent l'attitude, ici, de croire que seul un organismeà but non lucratif est à même de partager la mission et les objectifs des services publics. La société a but lucratif, qui devient partenaire à titre de délégataire d'une institution publique ou d'un gouvernement dans la gestion d'un service et le développement d'une ressource, fait de ces questions du partage des objectifs et de la qualité des services à la clientèle, le moteur même de ses activités et une des conditions de réussite de son entreprise, le profit ou le bénéfice en étant souvent la mesure.

De même, le délégateur public ne peut aller à l'encontre de l'essence même du partenaire qu'il entend choisir comme délégataire. Si c'est une société à but lucratif, le délégateur doit comprendre sa nature de réaliser une affaire rentable dans des conditions négociées. En souhaitant partager des risquesetbénéficier dessavoirfaire d'une entreprise, l'instance publique délégatrice doit être consciente que les conditions doivent être propices pour la survie et le succès de son partenaire. Sic'est une sociétéà but non lucratif, le délégateur doit tout autant en respecter la mission et les limites.

Il est done primordial que les deux partenaires réussissent leurs objectifs à travers l'expérience dans laquelle ils s'engagent et que des moyens appropriés soient adoptés pour ce faire. La volonté d'établir un véritable partenariat se fonde également sur un consensus pour collaborer et se concerter tout au long de la réalisation d'un mandat.

\section{Le respect du partenaire dans l'action}

Lorsque les objectifs préconisés par le délégateur sont clairement établis et étroitement liés à l'action, et lorsque les responsabilités sont précises, il devient beaucoup plus facile pour ce délégateur de laisser de la marge de manoeuvre à son partenaire privé dans le choix des moyens d'action. Le choix des moyens fait souvent partie d'une démarche conjointe où le délégateur peut profiter véritablement de l'expertise et des ressources privilégiées qu'il entend acquérir par cette association.

Les plans d'action sont déposés et discutés ouvertement. Des mécinismes adéquats sont prévus pour discuter des problèmes et des situations imprévus. C'est alors que l'onpeutvéritablementparlerdepartenariat fécondet d'une véritable synergie. Dans le cas où le délégateur se réserve le choix des moyens et la détermination de toutes les règles du jeu, on ne peut guère parler de partenariat, mais de délégation de gestion de façade.

Ainsi, lorsque les objectifs de performance sont élevés ou lorsqu'il s'agit d'un mandat à risque pour une société à but lucratif, le délégateur doit respecter l'expertise et la créativité de la ressource qu'il veut acquérir, sinon, pourquoi adopter des moyens qui vont peu à peu la scléroser? Le partenariat exige la confiance et il doit y avoir une place importante à l'expression de solutions novatrices.

\section{Conclusion}

Suite à notre expérience de plus de douze ans à titre d'entreprise privée, délégataire des pouvoirs publics au niveau de la prestation d'activités et de services aux usagers dans les parcs, nous croyons que le modèle de délégation de gestion que nous présentons pour les parcs provinciaux au Québec peut en être un d'avenir, permettant de re] ever les défis auxquels sont confrontés l'Etat québécoisetle milieu. Les conditions pour un partenariat efficace que nous soulevons nous semblent également, suite à notreexpérience, parfaitement applicables etoffrir, si elles sont respectées, desgaranties de succès pour un délégation de gestion dans les parcs du Québec. Nous espérons que les propos émis ici ajouteront à la réflexion qui se fait actuellement sur ces questions au Québec. 